\title{
EDITORIAL
}

\section{Aprendizaje Automático y Aprendizaje Profundo}

Actualmente hablar de Inteligencia Artificial (IA) es para algunos hablar de robots y películas de ciencia ficción tales como, Terminator, Matrix, Yo Robot entre otras, para otros es aún un tema de reflexión. Si bien existe alguna base común, donde en algún punto divergen hacia caminos distintos para la creación de este tipo de películas, la Inteligencia Artificial se focaliza en otros objetivos, como por ejemplo Aprendizaje Automático y Aprendizaje Profundo, por lo que entenderlas, nos dará una visión clara hacia donde se dirige hoy la Inteligencia Artificial.

Entender qué es la inteligencia artificial y cómo se puede emular fue un gran acontecimiento que se debe en gran parte a la contribución de Alan Turing. Gracias a la Prueba de Turing ${ }^{1}$, se logra tener una visión general de qué es la inteligencia. Turing definió el comportamiento inteligente como la capacidad de lograr un rendimiento a nivel humano en todas las tareas cognitivas, suficiente para engañar a un interrogador. Luego, el concepto formal de la IA fue acuñado en el Dartmouth Summer Research Project On Artificial Intelligence, en base a la propuesta de investigadores tales como John McCarthy, Marvin L. Minsky, Nathaniel Rochester y Claude E. Shannon, los cuales se reunieron para plantear las bases del aprendizaje y características de la inteligencia que pudiesen ser simuladas por una máquina ${ }^{2}$. Posterior a esto, Russell y Norving ${ }^{3}$ propusieron que el objetivo de la IA fuese generar máquinas que se comportaran como si fuesen inteligentes y se pudieran descomponer en diversas categorías, tales como sistemas que pensaran y actuaran como humanos y sistemas que pensaran y actuaran de manera racional. Además, estas máquinas pudieran realizar diversas tareas, tales como procesamiento de lenguaje natural, representación del conocimiento, razonamiento automático, aprendizaje automático, visión computacional y robótica, las cuales han marcado el caminar de la IA a través del tiempo.

El paso del tiempo, dió como resultado especializaciones y un tipo particular de IA llamado Aprendizaje Automático (AA), el cual fue definido por Samuel ${ }^{4}$ como el campo de estudio que da a los computadores la capacidad de aprender sin ser programados de manera explícita. Mitchell ${ }^{5}$, desde un punto de vista de ingeniería, lo define como un programa de computador que aprende de una experiencia $\mathrm{E}$, con respecto a una tarea $\mathrm{T}$ y una medida de rendimiento $\mathrm{R}$, si su rendimiento en $\mathrm{T}$, medido por $\mathrm{R}$, mejora con la experiencia E y luego Géron ${ }^{6}$ lo define como ciencia (y arte) de programar computadores para que aprendan a partir de los datos. Junto con estas definiciones, este tipo de IA ha ido creciendo con bastante rapidez debido a la cantidad de datos disponibles en la actualidad (Big Data) e Internet. Segundo a segundo crece la cantidad de datos para procesar, es por esto que se ha dividido en algunas categorías, tales como aprendizaje supervisado, aprendizaje no supervisado, aprendizaje por refuerzo, entre otras.

Si seguimos detallando el AA, llegamos al tipo de Aprendizaje Profundo (AP), donde su base son las Redes Neuronales Artificiales (RNA). La primera RNA conocida fue la propuesta por Mc Culloch

1 B.Y.A.M. Turing, "Computing Machinery and Intelligence by A.M . Turing 1 The Imitation Game 2 Critique of the New Problem". A M TURING, 1950.

2 J. McCarthy, M. L. Minsky, and C. E. Shannon. "A proposal for the Dartmouth summer research project on artificial intelligence - August 31, 1955". Ai Mag., 1955.

3 S. Russel and P. Norvig, Inteligencia Artificial: Un Enfoque Moderno, 1era ed. Prentice Hall, 1995.

4 A. L. Samuel, "Some Studies in Machine Learning," IBM J. Res. Dev., 1959.

5 T. M. Mitchell, "Does machine learning really work?," AI Mag., 1997.

6 A. Géron, Aprende Machine Learning con Scikit-Learn, Keras y TensorFlow, 2da ed. O’Reilly Media Inc., 2020. 
y Pitts ${ }^{7}$, la cual es un modelo computacional sencillo de cómo podrían trabajar juntas las neuronas biológicas en los cerebros animales para realizar computaciones complejas usando lógica proposicional. Así, Una RNA en AP, se diferencia en la cantidad de capas ocultas, típicamente en una RNA se puede encontrar una o más capas ocultas, pero en una RNA de AP puede tener cientos de capas ocultas, cada una con varias unidades de procesamiento distintas (neuronas). Desde la primera RNA hasta hoy en día, han crecido tanto los tipos y arquitecturas, como las problemáticas que estos tipos pueden resolver, por ejemplo, problemas de clasificación, visión computacional e interpretación de imágenes, procesamiento de lenguaje natural, patrones en datos, detección de objetos, reconocimiento de caracteres, etc. Así mismo, lo anterior puede ser aplicado en diferentes áreas, como minería, agricultura, economía circular, cambio climático, educación, etc.

Las RNA de AP más usadas en la actualidad son el Perceptrón Multicapa, las Redes Neuronales Convolucionales (CovNet), las Redes Neuronales Recurrentes y Redes Generativas Antagónicas. En particular las CovNet, gracias al trabajo de Yann LeCun, Yoshua Bengio y Geoffrey Hinton, ganadores del Premio Turing 2018, han experimentado una explosión en términos de sus usos y aplicaciones ${ }^{8}$. A esto se le suma el mayor protagonismo que han tenido las diferentes nuevas capacidades de cómputo, como nuevos hardware disponibles para cómputo de alto rendimiento, por ejemplo, las GPUs (Unidades de Procesamiento Gráfico). Además, no sólo se necesita una GPU para trabajar con Aprendizaje Profundo, sino también se puede trabajar de manera online, mediante Google Colab, Azure Microsoft, entre otros, permitiendo mejores y mayores avances en esta área.

Hoy en día, la Inteligencia Artificial, Aprendizaje Automático y el Aprendizaje Profundo están en una etapa de constante crecimiento, serán seguramente los ejes principales para el desarrollo de la ciencia de la computación y la humanidad donde estarán fundidas, no sólo hardware y software, sino también varias tecnologías, tales como nanotecnología, computación cuántica, automatización, entre otras. Así, la creación de nuevo conocimiento en torno a la Inteligencia Artificial ya sea de máquinas inteligentes, nuevos algoritmos, aprendizaje automático, aprendizaje profundo, entre otras, serán herramientas para acelerar la transición hacia una economía circular, donde se pueda diseñar productos y modelos de negocios circulares 9 .

En conclusión, el aporte de la IA, sus características, objetivos y tipos pueden ir de la mano con la evolución de la humanidad, transitando por un camino de mutuo beneficio.

\author{
MSc. Jorge Díaz-Ramírez \\ Universidad de Tarapacá \\ Departamento de Ingeniería y Tecnologías \\ Iquique, Chile \\ E-mail: jdiazr@academicos.uta.cl
}

\footnotetext{
7 W. S. McCulloch and W. Pitts, "A logical calculus of the ideas immanent in nervous activity," Bull. Math. Biophys., vol. 5, No 4, pp. 115-133, 1943.

8 Y. Lecun, Y. Bengio, and G. Hinton, "Deep learning," Nature. 2015.

9 McKinsey, "Inteligencia artificial y la economía circular: La IA como herramienta para acelerar la transición”, 2019. [En Linea]. Available: https://www.mckinsey.com/business-functions/sustainability/our-insights/artificial-intelligence-and-thecircular-economy-ai-as-a-tool-to-accelerate-the-transition/es-cl. [Accedido: 02-Oct-2020].
} 